\title{
Job Stress and Safety Climate in Cancer Treatment Centers: Upgraded Model for Dimensions
}

\author{
Saeed yari ${ }^{1}$, Saeed Shahsavari ${ }^{2}$, Mohammad Nourmohammadi ${ }^{3}$, Omid \\ Khosravizadeh $^{4}$, Ayda Fallah Asadi ${ }^{5}$
}

${ }^{1}$ School of Health Science, Shahid Beheshti University of Medical Sciences, Tehran, Iran. ${ }^{2}$ Health Products Safety Research Center, Qazvin University of Medical Sciences, Qazvin, Iran. ${ }^{3}$ Assistant Professor of Occupational Health Engineering Department, Health College, Mashhad University of Medical Sciences, Mashhad, Iran. ${ }^{4}$ Social Determinants of Health Research Center, Qazvin University of Medical Sciences, Qazvin, Iran. ${ }^{5}$ Nursing and Midwifery Department, Qazvin University of Medical Sciences, Qazvin, Iran.

\begin{abstract}
Background: Stress has always been with human despite all the progress in science and technology. Stressful conditions reduce concentration and lead to the lack of focus. This can increase human errors and bring about catastrophe. The aim of this study is to present a model to increase safety climate by controlling job stress among the personnel of Iran's medical centers. Methods: In this study, 680 personnel of Iran's hospitals were chosen in random. Demographic, job stress (35 questions) and safety climate (37 questions) questionnaires were filled. The data were inserted in SPSS 20 software. Descriptive statistics method and Pearson's correlation coefficient were used to describe the data and evaluate the relation between the variables, respectively. Results: The scores of safety climate and job stress were 3.40 and 3.21 , respectively which imply that they are suitable. The overall fitness of the model was acceptable. In this model, $\chi 2=6366.17, \mathrm{df}=2452, \chi 2 / \mathrm{df}=2.596, \mathrm{RMSEA}=0.054(90 \%$ $\mathrm{CI}=0.000-0.084), \mathrm{NFI}=0.901, \mathrm{GFI}=0.913$, and $\mathrm{CFI}=0.856$. In the regression analysis, there were positive significant relationships between job stress and safety climate, job stress and any of its components, and safety climate and any of the components. Conclusion: Job stress is directly related to safety climate. Since in this study job stress was in a suitable level, it influenced the safety climate. If job stress is high, then it can affect safety climate in a negative way. We suggest that further studies be done regarding this subject.
\end{abstract}

Keywords: Job stress- safety climate- patient safety- medicine personnel- modeling

Asian Pac J Environment and Cancer, 1 (1), 41-48

\section{Introduction}

In all organizations, especially the ones that offer people emergency services, an environment should be created in which no harm or damage threatens the employees. So, the safety of the hospitals as the most important organization offering medical services requires special attention. A hospital needs to be a safe place for patients and the personnel [1-3]. Safety climate is defined as a mental understanding of the personnel about organizational policies [4]. Recent studies indicate that there are significant relationships between safety climate and job stress [5]. But few studies have been done in this regard [6]. Stress has always been with human regardless of all the improvements in science and
Submission Date: 08/09/2018Ａcceptance Date: 10/08/2018

technology. No human has ever been able to free himself from stress. Organizations also have paid much attention to studying, controlling and reducing the effects of tress in work places to improve the efficiency of the employees [7]. So far more that 100 thousand articles and books have been publish about stress control and management and countries devote over 3 percent of their budgets to prevent stress. These indicate the importance of stress.

\section{Safety climate}

Safety climate is widely considered as a criterion for assessment of safety level in work places [8]. It presents an overall image of the safety situation of an organization [9]. Safety climate is multi-dimensional, but there is no consensus over the aspects of the safety climate [8-10].

Corresponding Author:

Dr. Ayda Fallah Asadi

Nursing and Midwifery Department, Qazvin University of Medical Sciences, Qazvin, Iran.

Email: asydafa@gmail.com 
Safety climate is defined as the common image workers have in mind about policies, processes, actions, and priorities in workplace [11-12]. This was first measured by Zoher et al. [12]. It is a measurement of the current state of the organization and it is relatively unstable based on time and place and changes due to circumstances [13]. So, safety climate is highly under the influence of organizational and personal elements. It might affect safety behaviors of the personnel [14]. Researchers also found out that the attitude of the workers regarding safety makes them search for safer environments, which in turn reduces unsafe behaviors and consequently improves safety situation [15-16]. Safety climate is defined as a psychological fundamental procedure in which workers share the quality of workplace [17]. Safety climate indicated that at the moment safety policies are in action and it can have a direct effect on safety behaviors of the workers. It can also predict future events [18-19]. Many researches on the other hand indicate that there is a robust connection between job accidents and the elements related to safety behaviors like job stress [20-21]. Stress is one of the subjects that need to be addressed in the area of safety management and organizational behavior [7].

\section{Job stress}

Job stress is an important subject in the 21 st century. It is proven to have effects on family, job and life quality [22]. If it not managed properly it affects the performance and health of the workers [10-23]. Job stress can be defined as harmful physical and emotional reactions; they occur when work job circumstances are not compatible with capabilities, resources and the workers' needs [22]. On the other hand, the personnel in organizations, and especially medical ones experience a high level of stress and psychological tension [24]. Job stress influences the health of people especially in cognitive, psychological, physiological and behavioral aspects. Also, in highly stressful situations, concentration decreases and ignorance increases [25]. This increases human error and leads to accidents [17]. These accidents include all types of mistakes and accidents in the hospitals [25]. Teachers, medical personnel, nurses and social workers, and emergency services are among the most stressed workers [24]. Nurses experience higher stress in comparison with other jobs [25-27]. The American National Association of Safety and Hygiene placed nursing at the top of 40 most stressful professions [28]. It is reported that $\% 93$ of the nurses are under constant stressful situations [29]. So, they probably suffer from cognitive impairment that may lead to safety accidents for the patients [25]. According to NIOSH, stress and psychological disorders are among the 10 major job damages in the US [30]. Every year about 1.1 million people lose their lives because of job stress and stress-related illnesses [7]. According to recent studies, counts for 50 to 60 percent of the lost days [31]. The Safety and Hygiene Bureau states (2004) that stress-related cost of society in England is $£ 3.7$ billion annually and job stress cost is about $£ 381$ million annually [32]. Stress also can lead to other behaviors like smoking and drinking [26]. Stress occurs when a human body is in an environment that is under constant change. Such changes leave physical and emotional effects and may provoke positive or negative feelings [33]. Job stress may vary from little to much, based on design, organization, job management, and social structure of the workplace [34-35]. Stressful circumstances are divided into intra- and extra-organizational groups [29]. Though there is generally a negative image regarding stress in the mind of people, human needs a balanced degree of stress to be able to operate efficiently. Without stress, people do not tend to make any effort to do anything. Some scholars believe that life without stress equals death. But too much stress leads to disorders and lack of efficiency [36-38]. Yet, job stress and its costs can be prevented [39]. This paper intends to offer a model to increase safety climate by controlling job stress among the personnel of Iran's hospitals.

\section{Materials and Methods}

\section{Design}

In this study, a descriptive analysis was performed on the personnel of Iran's hospitals. The main variables, here, job stress and safety climate; and personal and professional characteristics are considered as background variables.

\section{Data gathering tools}

Data gathering tools consisted of three questionnaires as follows:

A. Demographic information questionnaire: This part consisted of 9 questions including age, gender, marital status, education, major, job title, experience in the field, experience in the unit, and salary.

B. Job stress questionnaire: Job stress questionnaire included 35 questions, consisting of 5 alternative Likert scale (never, rarely, sometimes, often and always). Question scores indicate the measured amount for every item ranging from 1 to 5 in which 1 is unfavorable and 5 is the favorable state. This questionnaire includes 6 aspects of demand (8 questions), control (6 questions), support (9 questions), connection (4 questions), role (5 questions) and change (3 questions). Azad et al. studied the reliability and validity of the questionnaire for 749 military personnel of Iran. In their research the samples answered to HSE and GHQ questionnaire. Correlation coefficient of HSE and GHQ equaled $r=-0.48$. The results also indicate a strong correlation between the extracted elements from factor analysis and the aspects of the questionnaire. Validity of the questionnaire was 0.78 and .65 using Cronbach alpha and splitting methods. Finally the questionnaire was proved to be a reliable and credible one for studying job stress [40].

C. Safety climate questionnaire: This questionnaire consisted of 37 questions in form of Likert scale with 5 alternatives (quite disagree, disagree, no idea, agree and quite agree). Question scores indicate the measured amount for every item ranging from 1 to 5 in which 1 is unfavorable and 5 is the favorable state. This questionnaire includes 8 aspects of management commitment for safety and priority of safety matters (10 questions), 
the knowledge of the workers and following safety rules ( 7 questions), the attitude of the workers regarding safety (4 questions), cooperation of the workers and commitment to following safety (5 questions), safety of workplace (4 questions), priority of safety over products (2 questions), and neglecting dangers (2 questions). Zeidi et al. in a study through exploratory factor analysis showed that the 8 aspects of the questionnaire are able to explain 68.42 percent of the overall variance and Cronbach alpha coefficient for all the aspects and the whole of the tool was approved [41].

\section{Sampling method}

The studied population here was all the personnel of Iran's hospitals. The logic here was selecting 10 sampled for every variable according to James Stevens' proposal. He assumes that 10 to 15 items are required for each predictor variable, multiple regression analysis with the standard method of the least standard squares, as well as verifiable factor analysis models and structural equations [42-43]. Based on this 720 people were selected according to simple random sampling. Finally 680 questionnaires were filled and analyzed.

\section{Data gathering method}

The criteria for the participants entering the research were being volunteers and honesty. Then, they were assured that their answers were confidential and the data would be collected, evaluated and reported regardless of the names. Data gathering method was interview by the research team.

\section{Conceptual Model}

The aim of this study was evaluating the interaction between job stress and safety climate in Iran's hospitals. This model allows to study outside hidden variables (independent) and inside hidden variable simultaneously. The primary model and conceptual models are presented in Figure 1.

\section{Data analysis}

The gathered data were entered into SPSS 20 software. Descriptive statistics method was used to describe them. Also, Pearson's correlation coefficient was used to evaluate the relation between the variables. Structural Equation Modeling, which is a type of multivariate analysis, was used to evaluate the relation between job stress and safety climate [3]. Structural equations model was created using AMOS 22 software. The fitness of the model was controlled by $\chi 2$ and the root mean squares estimated error (RMSEA) was evaluated. The amount of $\chi 2$ ration to a degree of freedom smaller than $3(\chi 2 / \mathrm{df}<3)$ was chosen as a criterion to fit the model. RMSEA $<0.08$ was another criterion for the fitness of rhea model. Also, goodness of fit index (GFI), Comparative fit index, and normal fit index (NFI) were used.

\section{Ethical considerations}

Participants were informed about the nature of the study and were guaranteed confidentiality and anonymous presentation of the results. They were informed that their participation was voluntary, that they could withdraw from the study at any time. Written informed consent was obtained from all participants, to maintain anonymity and confidentiality during the entire process each consent was marked with a unique digit for each participant and the unique digit were kept during all stages of the research process.

\section{Results}

\section{Sample characteristics}

In the current study, among the 680 of the participants 422 were men $(\% 62), 258$ were women $(\% 38), 145$ (\%21.3) were single, $535(\% 78.7)$ were married, 104 (\%15.3) worked in the operation room, $104(\% 15.3)$ were anesthesiologists, $314(\% 46)$ were nurses, and $158(\% 24)$ had other positions. Also, majority of the participants (\%24) aged 31- 35 and had \%26.7 had 6-10 years of professional experience.

\section{Correlation coefficient}

Pearson's correlation coefficient for the measured variables is presented in Table 2. Job stress is directly related to the components of job stress (demand, control, support, communication, role, and change). Also, safety climate is directly related to the components of the safety climate (commitment, knowledge, attitude, participation, environmental safety, personal readiness, priorities, and ignorance). The mean of demand, control, support, communication, role and change, commitment, knowledge, attitude, participation, environmental safety, individual readiness, priority and ignore were 2.93, 3.16, $3.39,2.59,3.84,3.25,3.33,3.67,3.90,3.65,3.50,2.77$, 2.82 , and 290 respectively. Also the average safety climate and job stress were 3.40 and 3.21 respectively, both of which are appropriate.

In this study, gender $(\mathrm{p}<0.001)$, marital statues $(p=0.037)$, education $(p=0.008)$, position $(p=0.005)$, job experience $(p=0.045)$, work experience in the unit $(p=0.028)$, and salary $(p=0.031)$ were all significant in job stress. Also, gender $(\mathrm{p}=0.003)$, education $(\mathrm{p}=0.007)$, and position $(\mathrm{p}=0.042)$ were significant in safety climate.

\section{Structural equation modeling}

Figure 2 shows the coefficients of the standard

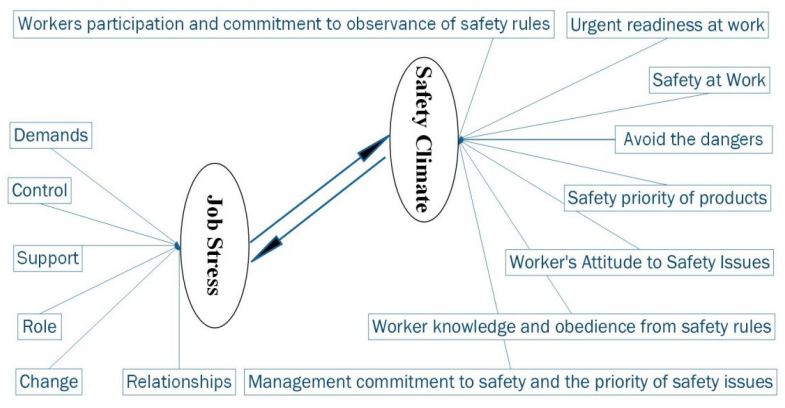

Figure. 1 Conceptual Model between Job Stress and Safety Climate 
Table. 1 Demographic Properties of Participants

\begin{tabular}{lccccccc}
\hline Variable & Group & Frequency & Percentage & Variable & Group & Frequency & Percentage \\
\hline Sex & Male & 422 & 62 & & Under 30 & 150 & 22 \\
& Female & 258 & 38 & & Between 31 and 35 & 163 & 24 \\
& Less than 5 & 177 & 26 & Age & Between 36 and 40 & 122 & 18 \\
& Between 6 and 10 & 182 & 26.7 & & Between 41 and 45 & 82 & 12 \\
& Between 11 and 15 & 86 & 12.7 & & Between 46 and 50 & 86 & 12.7 \\
Work experience & Between 16 and 20 & 100 & 14.7 & & Above 50 & 77 & 11.3 \\
& Between 21 and 25 & 72 & 10.7 & & Operating room & 104 & 15.3 \\
& Between 26 and 30 & 50 & 7.3 & \multirow{2}{*}{ Positions } & Anesthesia & 104 & 15.3 \\
& Above 30 & 13 & 2 & & Nursing & 314 & 46 \\
marital status & Single & 145 & 21.3 & & Others & 158 & 23.3 \\
& Marride & 535 & 78.7 & & & & \\
\hline
\end{tabular}

estimation of the structural equation model. All paths are at a significant level. However, according to the values obtained for fitting indexes in Table 3: $\chi 2 / \mathrm{df}$, GFI, CFI and NFI are not within the defined range. Therefore, it was concluded that fitting the model obtained at this stage does not indicate a good fit. So the model was corrected for a better fitting. These modifications were implemented in the proposed model and the results of the fitting indexes improved (Figure 3). Finally, the overall fitting of the model was acceptable. In this model: $\chi^{2}$ $=6366.17, \mathrm{df}=2452, \chi 2 / \mathrm{df}=2.596, \mathrm{RMSEA}=0.054$ $(90 \% \mathrm{CI}=0.000-0.084), \mathrm{NFI}=0.901, \mathrm{GFI}=0.913$, and $\mathrm{CFI}=0.856$.

In the regression analysis, job stress with safety climate, job stress with any of its components and safety climate with any of its components had significant relationships (Table 4).

Figure 2 shows the relationship between the components of the structural equations model. Job stress can be directly related to safety climate $(\beta=0.933, p<0.001)$. It is also directly related to demand $(\beta=0.899, p<0.001)$, control $(\beta=0.972, p<0.001)$, support $(\beta=0.923, p<0.001)$, communications $(\beta=0.740, p<0.001)$, and role $(\beta=0.825, p<0.001)$. Also, safety climate is directly

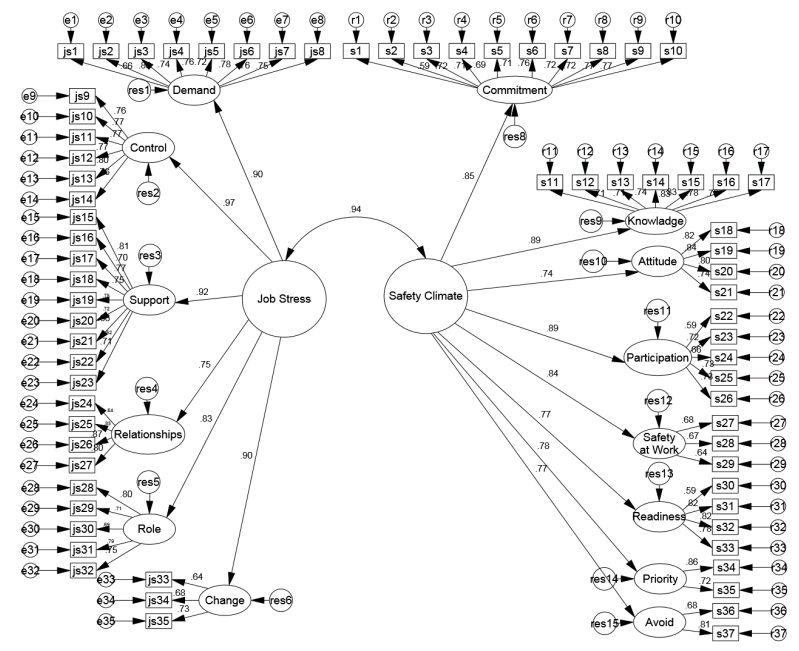

Figure 2. The Paths between the Components in the Proposed Model related to commitment $(\beta=0.819, \mathrm{p}<0.001)$, knowledge $(\beta=0.893, p<0.001)$, attitude $(\beta=0.697, p<0.001)$, cooperation $(\beta=0.867, p<0.001)$, safety of the environment $(\beta=0.850, p<0.001)$, personal readiness $(\beta=0.853$, $\mathrm{p}<0.001)$, and priority $(\beta=0.866, \mathrm{p}<0.001)$.

Also, according to the structural equation model, standard and non-standard coefficients of the final model and the significance level between variables and factors affecting it are presented in the following table.

\section{Discussion}

In a study conducted by Chen et al., it was found out that promoting a positive safety climate and creating educational programs could improve the safety performance of an organization due to improved mental health of employees, especially after traumas [10]. Park et al. found out that safety accidents of the patients are under the influence of job transfer, cognitive impairment and job stress. They also suggest that reduction in job instability and a definition of job limits is required for the patients' safety [25]. In a study done by Zarei et al. in medical organizations of Iran they concluded that there is a significant relationship between safety climate, unit type,

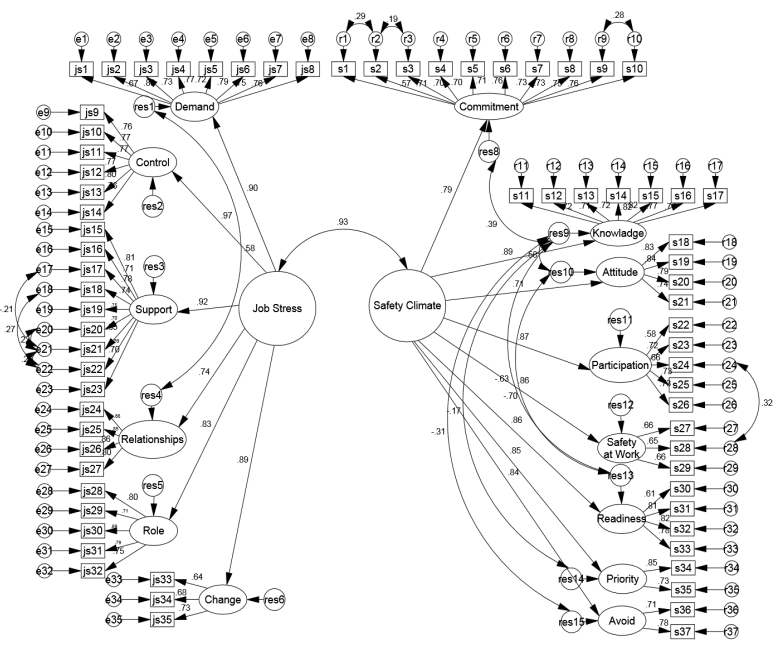

Figure 3. The Paths between the Components in the Final Model 


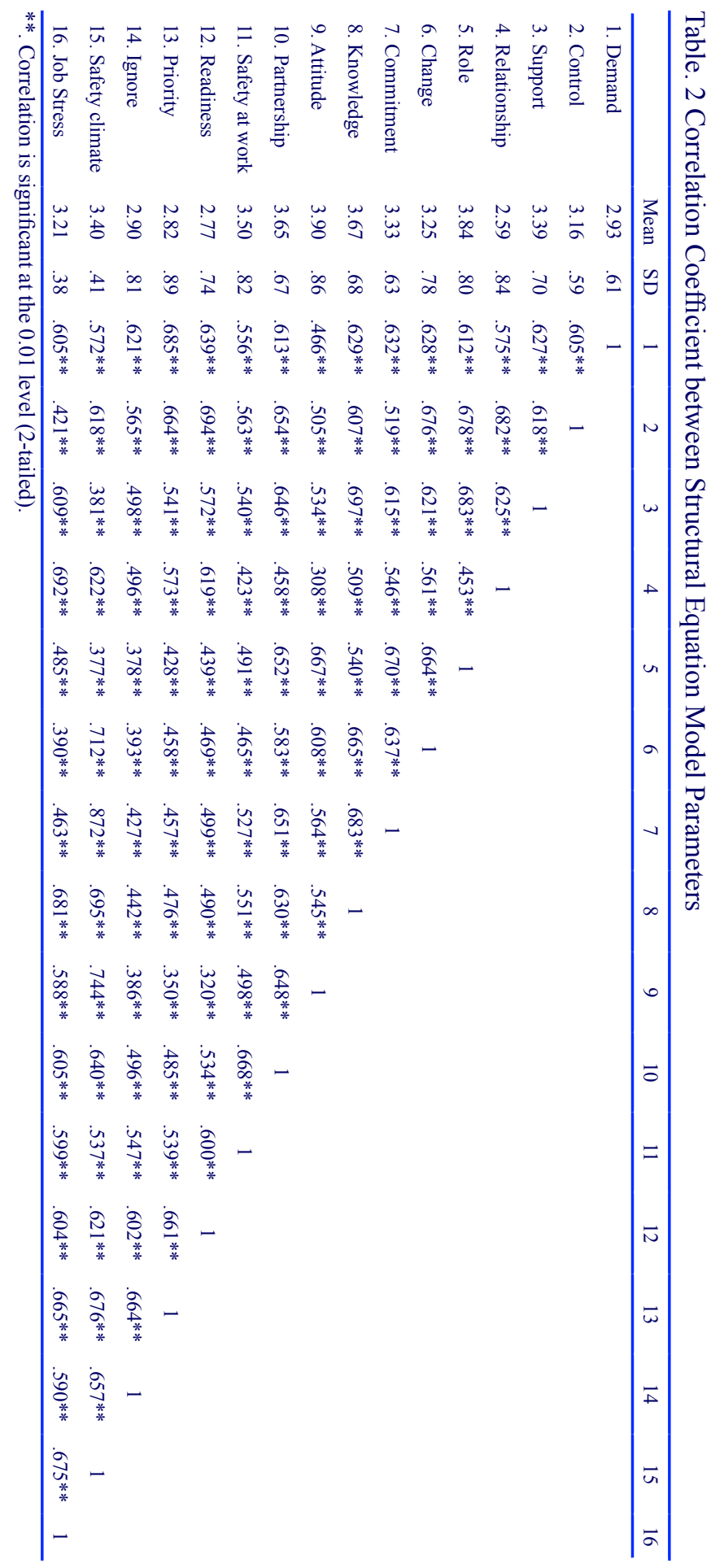

job satisfaction, job interests, and stress. The results of structural equation modeling support a negative correlation between job burnout and safety climate [44]. In this paper we concluded that the variables of gender, marital status, education, position, job title, work experience in the unit and salary had significant effects on job stress. Also, the variables of gender, education and position had significant effects on safety climate. In a study about the relationship between safety culture and occupational stress, Zamanian using independent t-test showed that there was a significant and negative relationship between these two variables [7]. Avram regards job satisfaction as a minor mediator between safety climate and organizational trust
[45]. Elshaer et al., in a study about occupational stress and burnout syndrome among health care workers, express that since there is a high stress and burnout among health care workers, more support is needed in this regard [24]. Hoboubi in a study calls for of corrective actions for shift jobs. He states that supervisor needs to offer support for job stress reduction and job satisfaction and productivity increase [35]. Kim addresses the importance of safety climate and psychological elements like job stress of the workers [17]. Yau's findings indicate that nurses have a relatively high level of stress, and "work environment and resources" and "workload and time" are known as stressors [46]. Dollard states that the safety climate can 
Table 3. Comparison of Fitness Indices in Conceptual Model and Proposed Model

\begin{tabular}{lccc}
\hline Index & Limit & conceptual model & proposed model \\
\hline$\chi 2 / d f$ & Less than 3 & 3.402 & 2.596 \\
GFI & Higher than 9.0 & .827 & 0.913 \\
RMSEA & Less than 08/0 & .09 & 0.054 \\
CFI & Higher than 9.0 & .827 & 0.875 \\
NFI & Higher than 9.0 & .861 & 0.901 \\
\hline
\end{tabular}

Table 4. Regression Weights in the Parameters of the Structural Equation Model in the Final Model

\begin{tabular}{|c|c|c|c|c|c|c|c|}
\hline & & & Standardized Estimate & Nonstandardized Estimate & SE & $\mathrm{CR}$ & P-value \\
\hline Job Stress & $<-->$ & Safety Climate & .933 & .500 & .043 & 11.618 & $* * *$ \\
\hline Control & $--->$ & Job Stress & .972 & 1.261 & .073 & 17.273 & $* * *$ \\
\hline Relationships & $--->$ & Job Stress & .740 & 1.015 & .068 & 14.984 & $* * *$ \\
\hline Role & $--->$ & Job Stress & .825 & 1.064 & .069 & 15.311 & $* * *$ \\
\hline Knowladge & $--->$ & Safety Climate & .893 & .936 & .063 & 14.955 & $* * *$ \\
\hline Attitude & $--->$ & Safety Climate & .697 & .826 & .058 & 14.347 & $* * *$ \\
\hline Priority & $--->$ & Safety Climate & .866 & .964 & .065 & 14.870 & $* * *$ \\
\hline Change & $--->$ & Job Stress & .890 & 1.000 & & & \\
\hline Demand & $--->$ & Job Stress & .899 & .903 & .060 & 15.012 & $* * *$ \\
\hline Commitment & $--->$ & Safety Climate & .819 & .613 & .049 & 12.620 & $* * *$ \\
\hline Support & $--->$ & Job Stress & .923 & 1.017 & .065 & 15.709 & $* * *$ \\
\hline Avoid & $--->$ & Safety Climate & .836 & 1.000 & & & \\
\hline Readiness & $--->$ & Safety Climate & .853 & .741 & .056 & 13.283 & $* * *$ \\
\hline Safety at Work & $--->$ & Safety Climate & .850 & .764 & .055 & 13.789 & $* * *$ \\
\hline Participation & $--->$ & Safety Climate & .867 & .655 & .051 & 12.762 & $* * *$ \\
\hline
\end{tabular}

***. Correlation is significant at the 0.001 level (2-tailed).

reduce job stress as an organizational feature and a proper climate enables the personnel to do their job [47]. Su states that hospital managers can help reduce work-related stress and improve the flexibility of tension among the nurses [48]. A study by Berland shows that the demand of a work environment with less control and social support from the colleagues increases stress, which can often affect patient safety [49]. Unlike the previous studies, the stress level among the staff working in hospitals in Iran was at an acceptable level. Job stress had a significant and positive relationship with the safety climate. In a study conducted by Idris et al. with the aim of a conceptual differentiation and its impact on occupational and mental health demand among Australian and Malaysian communities, it was found that the level of cognitive safety climate was significantly lower than the physical safety climate in both countries. This is an evidence of global lack of attention to mental health in working environments [39]. Understanding the professional perceptions of the patient's immediate safety climate in emergency service can help improve healthcare, reduce side effects and improve patient care through assessment of safety culture [50]. Mosadeghrad found out that organizational policy have strong bonds with job stress of the personnel. So, policies and hospital strategies need to change for the stress to decrease [51]. McLinton presents three methods used by managers and policymakers that can reduce social and psychological risks and bring about positive changes to the safety climate [52]. In a study by Ray et al. with the aim of employment arrangements, occupational stress and quality of life related to health they concluded employment arrangement played as an important predictor of occupational stress. Compared to stress-free individuals, people affected by employment arrangement have more unhealthy days or more activity restrictions [53]. MohammadFam believes that for the adjustment or removal of identified factors effective in creating stress and reducing unsafe actions, investments and stress management programs are needed [54]. Clarke argued that psychological depression has important impacts on safety outcomes, such as accidents and injuries [55]. Siu concluded that the tension in Western culture is not only about the West, but it seems to be a global problem [56]. Several other studies have strongly supported the links between worker safety and occupational stress and safety behaviors [57].

In conclusion, according to previous studies, in order to perform optimal activities, humans need a decent level of stress because without stress people do not make the necessary effort to do things. According to some scientists, life without stress equals death [36-38]. In this study, due to the fact that stress is favorable, it has increased the safety climate for the hospital staff. However, excessive stress, leads to impairment in performance and reduction of staff productivity. In the high stress societies, the relationship between occupational 
stress and safety climate can be significant and negatively affected. An increased stress can lead to a reduction in the safety climate. So, more research is needed in this area.

\section{Suggestions}

- One of the factors that may affect the results of this study is the sampling of all the staff working in the hospital. It is recommended that in future, studies focus on high stress societies, such as nurses.

- Considering that with an increase in the level of awareness job stress decreases, it is recommended that hospital managers make appropriate training in problem solving and stress management.

- To improve the level of patient safety in a hospital, job stress needs to be controlled and the climate and culture of safety should increase.

\section{Conflicts of interest}

All authors declare to have no financial or personal relationships that could inappropriately influence the research described.

\section{Acknowledgements}

Hereby we want to appreciate the cooperation of students, professors, hospital administrators, and all people in the sample who helped us throughout this study.

\section{References}

1. Yari S, Fallah AA, Varmazyar S. Assessment of semiquantitative health risks of exposure to harmful chemical agents in the context of carcinogenesis in the latex glove manufacturing industry. Asian Pacific journal of cancer prevention: APJCP. 2015;17(205):11

2. Yari S, Naseri MH, Akbari H, Shahsavari S, Akbari H. Interaction of Safety Climate and Safety Culture: A Model for Cancer Treatment Centers. Asian Pacific Journal of Cancer Prevention. 2007;20(3):961-9.

3. Yari S, Akbari H, Gholami Fesharaki M, Khosravizadeh O, Ghasemi M, Barsam Y, et al. Developing a model for hospital inherent safety assessment: Conceptualization and validation. International Journal of Risk \& Safety in Medicine. 2018 (Preprint): 1-12.

4. Radzaz NHBA, Bahari SF. Psychosocial safety climate in organization: An overview of theoretical and empirical development. Journal of Social and Development Sciences. 2013;4(9):407.

5. Law R, Dollard MF, Tuckey MR, Dormann C. Psychosocial safety climate as a lead indicator of workplace bullying and harassment, job resources, psychological health and employee engagement. Accident Analysis \& Prevention. 2011;43(5):1782-93.

6. Sampson JM, DeArmond S, Chen PY. Role of safety stressors and social support on safety performance. Safety Science. 2014;64:137-45.

7. Zamanian Z, Zakian S, Jamali M, Kouhnavard B. Relationship between Safety Culture and Job Stress among the Personnel of Telecom Companies. Safety Promotion and Injury Prevention. 2017;4(3):161-6.

8. Budworth N. The development and evaluation of a safety climate measure as a diagnostic tool in safety management. Iosh Journal. 1997;1:19-29.
9. Huang Y-H, Chen J-C, DeArmond S, Cigularov K, Chen PY. Roles of safety climate and shift work on perceived injury risk: A multi-level analysis. Accident Analysis \& Prevention. 2007;39(6):1088-96.

10. Chen Y, McCabe B, Hyatt D. Impact of individual resilience and safety climate on safety performance and psychological stress of construction workers: A case study of the Ontario construction industry. Journal of safety Research. 2017;61:167-76.

11. Griffin MA, Neal A. Perceptions of safety at work: a framework for linking safety climate to safety performance, knowledge, and motivation. Journal of occupational health psychology. 2000;5(3):347.

12. Zohar D. Safety climate in industrial organizations: theoretical and applied implications. Journal of applied psychology. 1980;65(1):96.

13. Lin S-H, Tang W-J, Miao J-Y, Wang Z-M, Wang P-X. Safety climate measurement at workplace in China: A validity and reliability assessment. Safety Science. 2008;46(7):1037-46.

14. Gatien B. An investigation into the relationship between perceptions of safety climate and organizational justice. 2010.

15. Yari S. Inherent safety design in compose of urban gas station. Safety Promotion and Injury Prevention. 2015;3(2):135-40.

16. Jafari MJ, Sadighzadeh A, Sarsangi V, Zaeri F, Yegani F. Safety climate survey in iran's uranium mines in 2013. Safety Promotion and Injury Prevention. 2014;2(3):148-54.

17. Kim KW, Park SJ, Lim HS, Cho HH. Safety climate and occupational stress according to occupational accidents experience and employment type in shipbuilding industry of korea. Safety and health at work. 2017;8(3):290-5.

18. Brown R, Holmes $H$. The use of a factor-analytic procedure for assessing the validity of an employee safety climate model. Accident Analysis \& Prevention. 1986;18(6):455-70.

19. Normohammadi M, Kakooei H, Omidi L, Yari S, Alimi R. Risk assessment of exposure to silica dust in building demolition sites. Safety and health at work. 2016;7(3):251-5.

20. Yari S. Assessment of potential risk by the failure mode and effects analysis in an air conditioning equipment manufacturing company. Safety Promotion and Injury Prevention. 2017;5(2):89-96.

21. Yari S, Pouyakian M, Jafari MJ, Alipour A, Varmazyar S. Preparation and psychometry of a safety assessment questionnaire for urban gas stations. Safety Promotion and Injury Prevention. 2018;5(3):169-80.

22. Azad-Marzabadi E, Fesharaki MG. Job Stress and Related Factors Among Iranian Male Staff Using a Path Analysis Model. Iranian Red Crescent Medical Journal. 2016;18(6).

23. Rafat M, Omid K, Tahere S, Hamed A. Job stress and its related factors in nurses of Qazvin University of Medical Sciences. The journal of Medical Education and Development. 2015;7(1):55-63.

24. Elshaer NSM, Moustafa MSA, Aiad MW, Ramadan MIE. Job stress and burnout syndrome among critical care healthcare workers. Alexandria Journal of Medicine. 2018;54(3):273-7.

25. Park Y-M, Kim SY. Impacts of job stress and cognitive failure on patient safety incidents among hospital nurses. Safety and health at work. 2013;4(4):210-5.

26. Johnson S, Cooper C, Cartwright S, Donald I, Taylor P, Millet C. The experience of work-related stress across occupations. Journal of managerial psychology. 2005;20(2):178-87.

27. Tourani S, Khosravizadeh O, Omrani A, Sokhanvar M, Kakemam E, Najafi B. The relationship between organizational justice and turnover intention of hospital nurses in Iran. Materia socio-medica. 2016;28(3):205.

28. Simmons BL, Nelson DL. Eustress at work: The relationship 
between hope and health in hospital nurses. Health care management review. 2001;26(4):7-18.

29. Parvin N, Kazemian A, Alavi A, et al. Evaluation nurses, job stressors in Shahrekord hospitals affiliated to medical university in 2002. 2005.

30. Quick JC, Macik-Frey M, Nelson DL. Job stress. 2016.

31. Golubic R, Milosevic M, Knezevic B, Mustajbegovic J. Workrelated stress, education and work ability among hospital nurses. Journal of advanced nursing. 2009;65(10):2056-66.

32. MacKay* CJ, Cousins R, Kelly PJ, Lee S, McCAIG RH. 'Management Standards' and work-related stress in the UK: Policy background and science. Work \& Stress. 2004.18(2):91-112.

33. Kawano Y. Association of Job-related Stress Factors with Psychological and Somatic Symptoms among Japanese Hospital Nurses: Effect of Departmental Environment in Acute Care Hospitals. Journal of Occupational Health. 2008;50(1):79-85.

34. Azad-Marzabadi E, Gholami Fesharaki M. Job Stress and Related Factors Among Iranian Male Staff Using a Path Analysis Model. Iranian Red Crescent Medical Journal. 2016;18(6):e34314. PubMed PMID: PMC5006310.

35. Hoboubi N, Choobineh A, Kamari Ghanavati F, Keshavarzi S, Akbar Hosseini A. The Impact of Job Stress and Job Satisfaction on Workforce Productivity in an Iranian Petrochemical Industry. Safety and Health at Work. 2017 2017/03/01/;8(1):67-71.

36. Doyle C. Work and organizational psychology: An introduction with attitude: Routledge; 2004.

37. Spector PE. Industrial and organizational psychology. Research and. 2008.

38. Stora JB. Le stress: Puf; 2016.

39. Idris MA, Dollard MF, Coward J, Dormann C. Psychosocial safety climate: Conceptual distinctiveness and effect on job demands and worker psychological health. Safety Science. 2012 2012/01/01/;50(1):19-28.

40. AZAD ME, GHOLAMI FM. Reliability and validity assessment for the HSE job stress questionnaire.2011.

41. Mohammadi Zeidi E, Farmanbar R, Hoseyni S. Assessment of Psychometric Properties (validity and reliability) of Safety Climate Questionnaire: Factor Analysis Application. Journal of Guilan University of Medical Sciences. 2012;21(81):1221. eng.

42. Byrne BM. Structural equation modeling with LISREL, PRELIS, and SIMPLIS: Basic concepts, applications, and programming: Psychology Press; 2013.

43. Chin WW, Peterson RA, Brown SP. Structural equation modeling in marketing: Some practical reminders. Journal of marketing theory and practice. 2008;16(4):287-98.

44. Zarei E, Khakzad N, Reniers G, Akbari R. On the relationship between safety climate and occupational burnout in healthcare organizations. Safety Science. 2016 2016/11/01/;89:1-10

45. Avram E, Ionescu D, Mincu CL. Perceived safety climate and organizational trust: the mediator role of job satisfaction. Procedia-Social and Behavioral Sciences. 2015;187:679-84.

46. Yau SY, Xiao XY, Lee LYK, Tsang AYK, Wong SL, Wong KF. Job stress among nurses in China. Applied Nursing Research. 2012 2012/02/01/;25(1):60-4.

47. Dollard MF, Tuckey MR, Dormann C. Psychosocial safety climate moderates the job demand-resource interaction in predicting workgroup distress. Accident Analysis \& Prevention. 2012.694:45-704.

48. Su S-Y, Chiou S-T, Huang N, Huang C-M, Chiang J-H, Chien L-Y. Association between Pap smear screening and job stress in Taiwanese nurses. European Journal of Oncology Nursing. 2016 2016/02/01/;20:119-24.
49. Berland A, Natvig GK, Gundersen D. Patient safety and job-related stress: A focus group study. Intensive and Critical Care Nursing. 2008 2008/04/01/;24(2):90-7.

50. Rigobello MCG, Carvalho REFLd, Guerreiro JM, Motta APG, Atila E, Gimenes FRE. The perception of the patient safety climate by professionals of the emergency department. International Emergency Nursing. 2017 2017/07/01/;33:1-6.

51. Mosadeghrad AM, Ferlie E, Rosenberg D. A study of relationship between job stress, quality of working life and turnover intention among hospital employees. Health Services Management Research. 2011 2011/11/01;24(4):17081.

52. McLinton SS, Dollard MF, Tuckey MMR. New perspectives on psychosocial safety climate in healthcare: Amixed methods approach. Safety Science. 2018 2018/11/01/;109:236-45.

53. Ray TK, Kenigsberg TYA, Pana-Cryan R. Employment arrangement, job stress, and health-related quality of life. Safety Science. 2017 2017/12/01/;100:46-56.

54. Mohammadfam I, Bahrami A, Fatemi F, Golmohammadi R, Mahjub H. Evaluation of the Relationship between Job Stress and Unsafe Acts with Occupational Accidents in a Vehicle Manufacturing Plant. Avicenna Journal of Clinical Medicine. 2008;15(3):60-6. eng.

55. Clarke S. An integrative model of safety climate: Linking psychological climate and work attitudes to individual safety outcomes using meta-analysis. Journal of Occupational and Organizational psychology. 2010;83(3):553-78.

56. Siu O-1, Phillips DR, Leung T-w. Safety climate and safety performance among construction workers in Hong Kong: The role of psychological strains as mediators. Accident Analysis \& Prevention. 2004 2004/05/01/;36(3):359-66.

57. Strahan C, Watson B, Lennonb A. Can organisational safety climate and occupational stress predict work-related driver fatigue? Transportation Research Part F: Traffic Psychology and Behaviour. 2008 2008/11/01/;11(6):418-26.

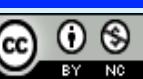

This work is licensed under a Creative Commons AttributionNon Commercial 4.0 International License. 